NAI PROPOSAI NO. 191

Scientific Spokesman:

Charles D. Orth

Building 46

Lawrence Berkeley Laboratory

University of California

Berkeley, California 94720

FTS/Comm: (415) 843-2740 ext. 5235

Total Absorption Shower Counter Test

C. D. Orth, G. F. Smoot, I. H. Smith

A. Buffington, R. A. Muller, P. M. Dauber

Space Sciences Laboratory, University of California, Berkeley, California

R. J. Kurz

Instrument systems Department, TRW Systems Group, Redondo Beach, California 
NAL EXPERIMENTAL PROPOSAL

3 November 1972

\section{Title: Total Absorption Shower Counter Test}

Abstract: We propose to conduct a short test to check the basic concepts used in the Total Absorption Shower Counter (TASC) being developed for the Alvarez cosmic-ray experiment (BCR-5) on the HEAO-B satellite. The objective is to ascertain whether the probability for an incoming proton of energy $E \geq 100 \mathrm{GeV}$ to interact in the TASC and simulate the response due to an incoming $e^{ \pm}$is smaller than $10^{-4}$. We will thereby obtain a preliminary estimate of whether the TASC should be able to

distinguish incident $\mathrm{e}^{ \pm}$from incident protons to one part in $10^{4}$ over the $B C R-5$ momentum range from 5 to $10^{4} \mathrm{GeV} / \mathrm{c}$.

\section{Experimenters:}

Space Sciences Laboratory, University of California, Berkeley, California Dr. Charles D. Orth

Dr. George F. Smoot

Dr. Lawrence H. Smith

Dr. Andrew Buffington

Dr. Richard A. Muller

Dr. Philip M. Dauber

Instrument Systems Department, TRW Systems Group, Redondo Beach, California

Dr. Richard J. Kurz

Scientific Spokesman: Dr. Charles D. Orth Building 46 Lawrence Berkeley Laboratory University of California

Berkeley, Ca. 94720

Phone: (415) 843-2740, ext. 5235 


\section{PHYSICS JUSTIFICATION}

We here propose to run a short test to check the basic concepts used in the design of the Total Absorption Shower Counter (TASC) being developed for the Alvarez cosmic-ray experiment on the HEAO-B satellite (this satellite experiment will be referred to hereafter by its official NASA designation, $B C R-5)$. This satellite experiment utilizes a superconducting magnetic spectrometer for separate measurement of cosmic-ray electron and positron spectra, as well as nuclear and anti-nuclear spectra ( $\mathrm{H}$ to $\mathrm{Fe}$ ), as a function of (momentum/charge) above geomagnetic cutoff. A TASC is included to measure the energy of the electrons and positrons and to provide the means for distinguishing incident $\mathrm{e}^{ \pm}$events from incident proton events. The primary objective for BCR-5 is to determine the positron contribution to the combined electrontpositron flux, and hence determine the relative contributions from primary and secondary electron source mechanisms. Experiment objectives require that the TASC provide the means to distinguish incident $\mathrm{e}^{ \pm}$'s from a background of protons to one part in $10^{4}$ over the momentum range from 5 to $10^{4} \mathrm{GeV} / \mathrm{c}$. The extent to which this separation can be accomplished will determine the degree of success of the BCR-5 experiment. According to our Monte Carlo calculations, the limiting factor in $\mathrm{e}^{ \pm}$proton discrimination is the probability that a proton interacts in the first few radiation lengths of the TASC and concentrates nearly all of its energy into neutral-pion production. These neutral pions then decay and initiate an electromagnetic shower indistinguishable from that initiated by an $\mathrm{e}^{ \pm}$of comparable energy. Such an interaction is not common, since a proton interaction generally concentrates most of the incident energy in the scattered proton and charged hadronic secondaries. It is impossible to compute accurately how likely such a process is with the present lack of understanding of strong-interaction dynamics and lack of sufficient 
data on proton-nucleus interactions in heavy absorbers at high energies. A complicating factor is that the resulting simulation of $\mathrm{e}^{+}$responses is something which occurs to varying degrees and is dependent on the particular instrument used; the simulation might be quite imperfect on theoretical grounds and still appear perfect depending on the design and data-analysis techniques employed to distinquish different particle types in that instrument.

To maximize the quality of the information our TASC would provide regarding the type of incident particle, we chose a design with the following major features. The bulk of the material is tungsten, which gives a maximum ratio of radiation lengths to proton interaction lengths for a material which is reasonably easy to handle. The total depth of the instrument is twenty radiation lengths, which is sufficient to contain $\mathrm{e}^{ \pm}$showers but usually not sufficient to absorb more than a small fraction of the energy of an incident proton. Energy sampling is accomplished every radiation length to maximize the information provided on the development of a shower. Once the design is fixed, the crux is to determine the data-analysis criteria for the characterization of a particular event as one due to an $e^{ \pm}$or a proton. We assume that responses for $e^{t \prime} s$ can be reliably determined through Monte Carlo calculations with experimental verification at SLAC energies. Reliable proton responses, on the other hand, must be determined empirically because the proton interactions of interest here are rather improbable. Nevertheless, in an effort to assemble a preliminary set of triggering and data-analysis techniques for the separation of electrons and protons at high energies, we have made Monte Carlo predictions for both electrons and protons based on extrapolation of low-energy data for our TASC. 


$$
-3-
$$

One trigger criterion which we formulated is the requirement that every event accepted produce at least four times minimum ionization is a scintillator located at a depth of two radiation lengths in the TASC. Such a requirement is expected to reject four-fifths of the incident protons, but less than several percent of the incident $e^{t_{1}} s$.

What is now required is an experimental check to verify the feasibility of these design concepts and analysis techniques. It is for this reason that we are proposing to run the short test described on the following pages. 


\section{EXPERIMENTAL ARRANGEMENT}

This test requires an exposure of our apparatus to a beam of protons at a single energy greater than or equal to $100 \mathrm{GeV}$. After a setup time of about one week, there will be one exposure lasting approximately 14 hours. The first part of this exposure will allow us to investigate the size of the proton interaction length, the fraction of protons meeting the trigger requirement of four times minimum ionization at a depth of two radiation lengths, and so forth. In addition, this investigation is expected to provide a quick on-line estimate of whether protons can simulate electrons to one part in $10^{4}$ by measuring the ability of protons to produce the large responses expected near shower maximum for electrons of the same energy. The second part of the exposure will be the main data run in which we shall record $10^{4}$ proton events meeting the four times minimum ionization trigger requirement. Because our data handling electronics limits our rate of data accumulation to one event every 22.5 milliseconds, and each event must be isolated from any other event by at least 100 nanoseconds, a possible choice of experimental area for these runs would seem to be somewhere along the 30" $\mathrm{HBC}$ line (beam N3). Therefore, to be specific, we shall discuss our experiment with this location in mind. We shall assume that the composition of the beam has already been measured and meets the criteria shown in Table 1.

TABLE 1

MAXIMUM PERMISSIBLE BEAM CONTAMINATIONS

Contaminant

$$
\frac{e^{+}}{\mu^{+}}{ }^{+}
$$

Maximum Allowed Ratio to Proton Flux

$$
\begin{gathered}
\text { LARGE } \\
\sim 1 \\
\sim 0.1 \\
\sim 1
\end{gathered}
$$


If these measurements have not been made, we would expect to make them ourselves before our exposure. In the event that the $\pi^{+}$or $\mathrm{K}^{+}$contamination is larger than that shown in Table 1, we will require use of the NAL Cerenkov counter located between enclosures 105 and 106 to tag these particles during our data run.

Knowledge of the beam composition is critical for our test because of the rarity of the process we are trying to observe. Because this process involves the simulation of an electromagnetic shower, positron contamination must definitely be less than $10^{-4}$. We have indicated in Table 1 that a "1arge" positron contamination is permissible only because we plan to use an extremely efficient technique for the rejection of $e^{+}$events. This technique requires no larger than minimum-ionizing signals in a scintillator placed directly behind a thick lead absorber. Because showers initiated by 100 to $300 \mathrm{GeV}$ positrons reach their maximum development at a depth of 8 to 10 radiation lengths, effective $e^{+}$screening can be accomplished by using a lead absorber of just this thickness. Although some incoming protons will interact in this much lead and thereby be rejected, this thickness is not large enough to result in any significant inefficiency in triggering on proton events.

Large $\mathrm{K}^{+}$and especially large $\pi^{+}$contaminations are intolerable because their charge exchange reactions can result in neutral pion production in

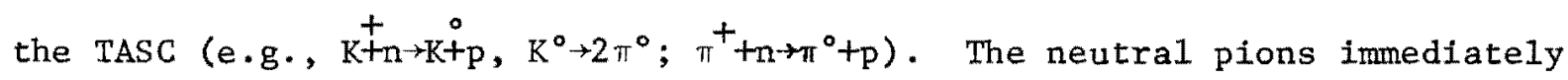
decay into photons which initiate electromagnetic showers. In order to keep these processes below the $10^{-4}$ leve1, we require the $\mathrm{K}^{+}$and $\pi^{+}$contaminations to be smaller than the maximum allowable contaminations shown in Table 1. These maximum allowable contaminations were estimated as follows: 
$\mathrm{K}^{+} / \mathrm{p}<10^{-4} \times$ (Fraction of protons not interacting in lead)

/ (Chance of $\mathrm{K}^{+}$not decaying in 1000 meters from hadron target)

/ (Chance of $\mathrm{K}^{+}$not interacting in lead)

/ (Chance of $\mathrm{K}^{+}$interacting in first two radiation lengths of TASC)

/ (Chance interaction makes a $\mathrm{K}_{\mathrm{S}}^{0}$ )

/ (Chance of $\mathrm{K}_{\mathrm{S}}^{\circ}$ decaying somewhere in TASC)

I (Chance the decay mode was $\mathrm{K}_{S}^{\circ} \rightarrow 2 \pi^{\circ}$ )

$$
\approx \frac{10^{-4} \times(3 / 4)}{(.3 \text { to } .5)(3 / 4)(0.05)(1 / 10)(1 / 10 \text { to } 1 / 5)(1 / 3)}
$$

$\approx$ unity

$\pi^{+} / \mathrm{p}<10^{-4} \times$ (Fraction of protons not interacting in lead)

/ (Chance of $\pi^{+}$not decaying in 1000 meters from hadron target)

$/$ (Chance of $\pi^{+}$not interacting in the lead)

$/$ (Chance of $\pi^{+}$interacting in first two radiation lengths of TASC)

/ (Chance that interaction was charge exchange)

$$
\approx \frac{10^{-4} \times(3 / 4)}{(.8 \text { to } .9)(3 / 4)(0.06)(0.02)}
$$

$\approx 1 / 10$

These contamination limits are those immediately after the hadron target, and are computed for an experimental site assumed to be at the 30 " $\mathrm{HBC}$ and a beam energy in the neighborhood of 100 to $200 \mathrm{GeV}$. Note that we have used the fact that the lead absorber constitutes one-fourth of one proton interaction length (one proton interaction length in lead is about 194 $\mathrm{g} / \mathrm{cm}^{2}$ ), so only about three-fourths of the incoming protons traverse the lead without interaction.

A muon impurity would be a nuisance while attempting to investigate 
the interaction properties of protons during the first part of our exposure. We sha11 assume, however, that any muon contamination which exists while the pi-plus contamination is below $10 \%$ will be acceptable.

To assess the time required for data accumulation, we sha11 assume that no beam time will be needed for beam-composition studies. For the first part of our exposure, we shall use scintillation counters alone or use a trigger rate low enough that deadtime in the electronics will be essentially nil. We will then be able to run with a higher beam intensity. To make the run as short as possible, we require a beam intensity higher than about 40 particles per 100-microsecond pulse occurring every 4 seconds, or, we shall conduct this run and the main data run simultaneously. If the higher beam intensity can be achieved, over $10^{4}$ protons can be examined in under two hours of beam time during this run.

In the main data run, we want to accumulate the equivalent of $5 \times 10^{4}$ proton events. We shall here be severely limited by deadtime in the electronics, which is 22.5 milliseconds per recorded event $(720$ bits at $32 \mathrm{k}$ bits/second). The maximum rate of data accumulation will hence be one event per 100-microsecond pulse. For triggering, we shal1 require greater than four times minimum ionization in the scintillator after the second radiation length in the TASC, just as we plan to do in our satellite experiment. Although on $1 y$ $6 \%$ of the protons are expected to interact in the first two radiation lengths (one proton interaction length in tungsten is about $188 \mathrm{~g} / \mathrm{cm}^{2}$ ), backscattering from interactions deeper in the TASC make about $1 / 5$ of the protons meet the four-times-minimum-ionization trigger requirement. We therefore expect to record $10^{4}$ events in this run. With approximately ten particles per pulse (or more), we might hence expect to complete 
the run in about $10^{4}$ pulses, provided beam fine structure doesn't hamper the data taking. Beam fine structure could be a problem if main-ring bunching at 20-microsecond intervals prevents each recorded event from being isolated from any other event by at least 100 nanoseconds. This isolation is required because our electronics uses 100-nanoseconds-width gates for 24 essential $\mathrm{ADC}^{\prime} \mathrm{s}$.

Assuming that beam fine structure will not be a problem, we estimate the main data run will require between 11 and 12 hours of beam time. The entire test would then be completed in less than 14 hours of beam $t$ ime. The equipment we plan to use for these runs is shown schematically in figure 1. The basic beam telescope is comprised of two 1/4-inch-thick scintillators, S1 and S2, which limit the accepted beam size. Anticoincidence scintillator $\mathrm{A} 2$, which has a hole the size of $\mathrm{S} 2$, is included to eliminate triggering on stray particles, beam halo, and some of the protons which have interacted in the lead absorber. This lead absorber, $4.5-\mathrm{cm}$. thick ( 8 radiation lengths), is followed by a $1 / 4$-inch-thick anti-coincidence scintillator $A 1$ whose function is to veto all beam particles infiating an electromagnetic shower in the lead. The beam telescope is followed by the TASC Model, which is constructed of 25 one-radiationlength-thick tungsten plates separated by 3/8-inch slots.* These slots are

* Although the BCR-5 TASC is 20 radiation lengths deep, we shall here use a slightly deeper TASC to investigate the merits of larger depths. The difference should not be significant, however, because most electromagnetic showers are absorbed in 18 to 20 radiation lengths. 

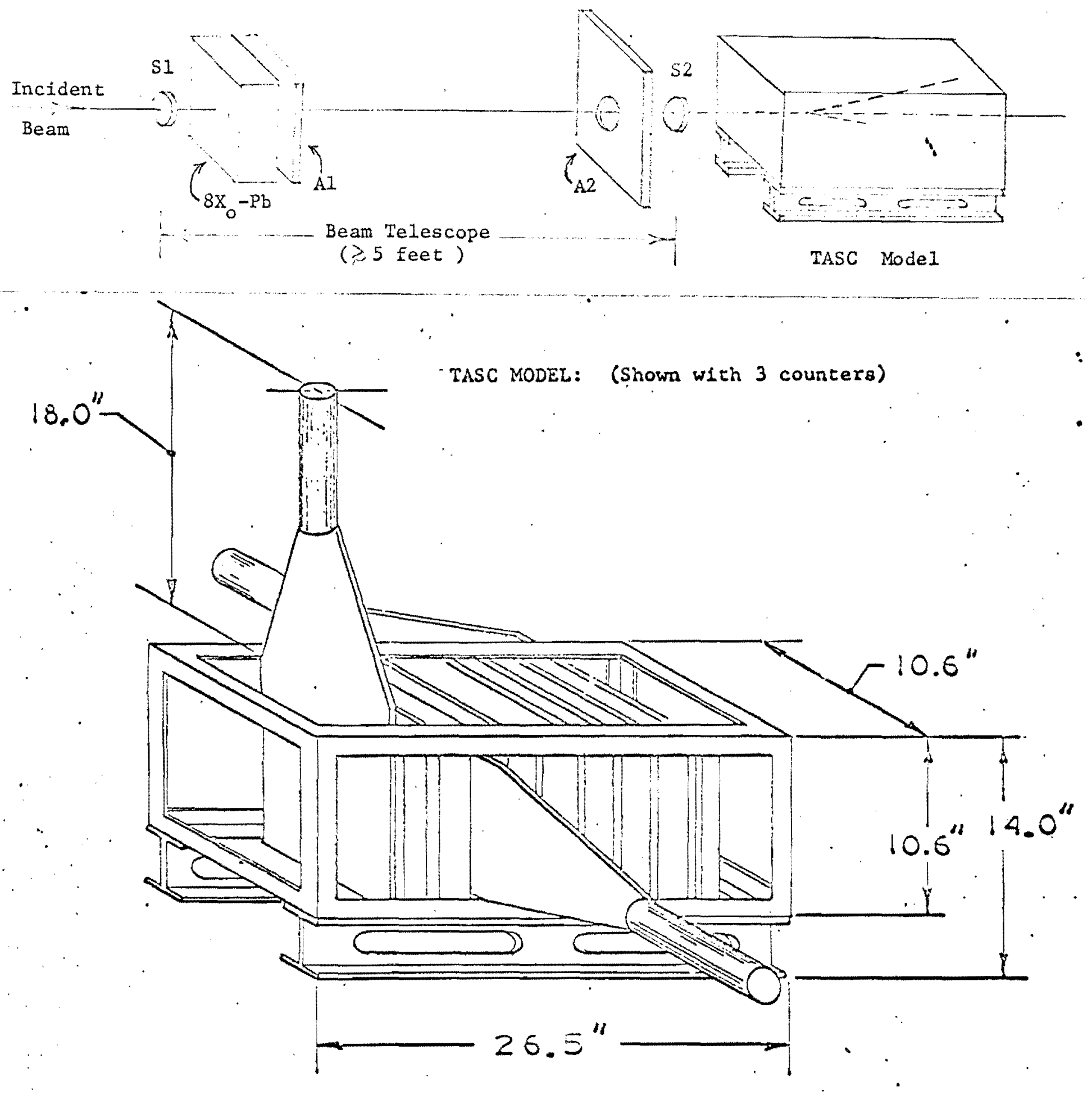

25 Tungsten layers each $0.14 \times 9.75 \times 9.75$ in.

22 Pilot-Y Scintillators each $0.25 \times 9.75 \times 9.75$ in. with RCA 4523 or RCA 8575 photomultiplier tubes

2 Multiple-wire proportional chambers each $0.3 \times 9.75 \times 9.75$ in. FIGURE 1: EXPERTMENTAL APPARATUS 
filled in a specific order with twenty-two 1/4-inch-thick plastic scintillation counters to sample energy deposition and two narrow-gap multiplewire proportional chambers to sample lateral shower spread (see figure 1 for construction details). The TASC complete with detectors, 1ightpipes, and photomultiplier tubes fits inside a volume approximately $47^{\prime \prime}$ wide by $32^{\prime \prime}$ high by $27^{\prime \prime}$ 1ong. The active detector area is about $9 " \times 9^{\prime \prime}$. 
III. APPARATUS:

The equipment required for this proposed test is listed below. The only item not now ready for shipment to NAL is the TASC Model complete with assorted detectors, but it will be ready by mid-November 1972 . The power required for the electronics is 30 amperes single phase at 110 volts. The manpower which we will supply for the test consists of at least three physicists on duty at all times plus one electronic engineer or technician as required.

Equipment

1. TASC Mode1 with scintillation and Cerenkov detectors, lightpipes, and photomultiplier tubes

2. Two multiple-wire proportiona1 chambers

3. Fast logic and datareadout electronics

4. Beam telescope

5. Three wire spark chamber planes with magnetostrictive readout *

6. Differential-threshold Cerenkov counter for $\pi-p$ separation for $30 "$ $\mathrm{HBC}$, complete with signal cables to our experimental site.

7. Two movable extension carts
Cost/Worth New/Existing

Space Sciences Lab (Berkeley)

$\$ 10 \mathrm{~K}$

New

$\$ 20 \mathrm{~K}$

Existing

TRW

NASA (MSC)

$\$ 300 \mathrm{~K}$

Existing

Space Sciences Lab

(Berkeley)

NASA (MSC)

$\$ 1 \mathrm{~K}$

$\$ 10 \mathrm{~K}$

Existing

NAL

L.S.U.

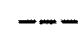

Existing

\section{New Costs $=\$ 17 \mathrm{~K}$ \\ Existing Equipment $=\$ 330 \mathrm{~K}$}

